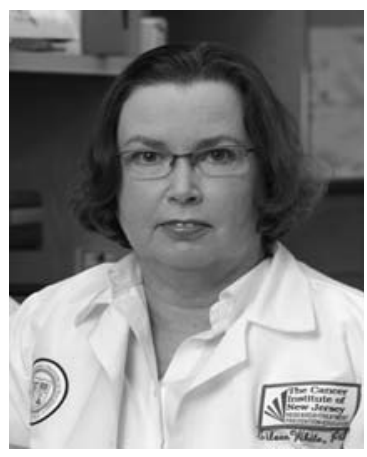

\title{
A Conversation with Eileen White
}

\author{
INTERVIEWER: ElizABeth McKenNA \\ Associate Editor, Cancer Discovery
}

Eileen White is a Distinguished Professor of Molecular Biology and Biochemistry at Rutgers University and the Deputy Director and Associate Director for Basic Science at the Rutgers Cancer Institute of New Jersey.

Elizabeth McKenna: Could you explain what autophagy is?

Dr. White: It's a very interesting process where, in response to starvation, cells will make vesicles that will capture components in their cytoplasm-mostly proteins and organelles - and then degrade them. The idea is that this degradation of proteins and organelles provides breakdown products that can be recycled into metabolic pathways. In that way, cells in animals can tolerate starvation for short periods of time. It's a survival pathway first identified in yeast that allows yeast to survive nitrogen starvation, and the use of autophagy to survive starvation is also apparent in mammals. As a matter of fact, we made a mouse where we can systemically, acutely delete an essential autophagy gene. Those mice are okay in the short term, but if you fast them, they die.

Clearly, autophagy's a survival pathway in yeast that's conserved all the way to mammals. What's interesting is that this pathway is normally on only at a very low level under normal conditions, but it's massively induced when cells or animals are starved. What was unusual is that when you look at cancer, even under fed conditions, the autophagy pathway appears to be on. When we interfered with the function of the autophagy pathway, this diminished the growth and survival of cancer cells. What that meant was that autophagy was a survival pathway for normal cells, but cancer cells can usurp or take advantage of that pathway for their own survival.

Elizabeth McKenna: You've been working on the role of autophagy in K-RAS-driven cancers. Can you tell us what you've found? Is there a specific way that autophagy is promoting survival in K-RAS-driven cancers?

Dr. White: I'm really working on two things. One is: What specifically is autophagy doing, and does it in fact recycle intracellular components? If so, what metabolic pathways are supported by this recycling? The first step is, does recycling of intracellular components occur in cancer cells? We did this by demonstrating that labeling components in cancer cells, and then starving the cells, caused the label to start appearing in an autophagy-dependent manner into specific metabolic pathways. We were able to do that. In fact, proteins and organelles are degraded by the process of autophagy, but if you delete an essential autophagy gene that process does not occur efficiently. Cancer cells are recycling macromolecules by autophagy.

The second question is: What are they using it for and how does it promote survival? The short answer is that they need autophagy to prevent fatal nucleotide pool depletion. When you starve RAS-driven cancers, if they don't have autophagy, they undergo an energy crisis and they deplete their pools of nucleotides. What autophagy is doing is supplying substrates to maintain energy, nucleotide synthesis, and nucleotide pools.

Elizabeth McKenna: Does autophagy work to promote survival in the same way in different cancers with different genetic backgrounds, like B-RAF for example, or is it a different mechanism?

Dr. White: I don't know that we know the answer to that. These metabolic interrogations of RAS-driven cancers that were wild type and knocked out for autophagy have only been done in RAS-driven lung cancer. We have not yet done them in other cancers. What we know is that there are probably multiple functions of autophagy that are contributing to cancer. For example, in B-RAF-driven melanoma models, autophagy prevents senescence. That could really be related to a metabolic defect caused by the loss of autophagy, but we'll have to see.

Elizabeth McKenna: Do you suspect that it's a difference based on the genetics or based on just the nature of different tissues?

Dr. White: I think the genetics is going to make a difference, but I think the tissue type might also make a difference. We'll have to see.

(C) 2016 White. This article is distributed under the terms of the Creative Commons Attribution-NonCommercial License, which permits reuse and redistribution, except for commercial purposes, provided that the original author and source are credited. 
Elizabeth McKenna: Other people in the field have found that, in certain contexts, autophagy can actually be tumor-suppressive. Can you comment on that?

Dr. White: We have published papers related to that too. The take-home lesson is that autophagy has a contextdependent role in cancer. In some cancers, autophagy promotes survival like it does in yeast and mammals during starvation. In some cases, autophagy does nothing; there are cancer cells that don't care if autophagy is there or not. That's interesting, because how are these cells dealing with stress if they don't have autophagy? That's an important future area for investigation. In other models, if you knock out autophagy, especially in the liver, you end up with benign hepatomas. It's clear that in mouse models autophagy is suppressing the development of benign liver tumors. One possibility, and I think this is generally what people are thinking, is that autophagy protects cells. It promotes their survival, but in tissues like the liver where chronic damage and cell death leads to chronic inflammation - and chronic inflammation in the liver and pancreas and a few other tissues is a wellknown carcinogen - that it is probably the mechanism whereby autophagy is tumor-suppressive. This is occurring in mouse models, and it's not clear whether this occurs in humans.

Elizabeth McKenna: Can you tell us about some of the efforts to characterize this in humans?

Dr. White: One key indicator of anything in cancer is simply to look at its genome. Right now, more than 10,000 human tumors have been sequenced. We have a huge amount of data on genomic alterations in a vast number of genes. Essentially, the genetic footprint of whether or not these genes are involved in cancer can be seen by looking at this data. What we've learned is that, in general, the core autophagy machinery is not mutated in cancer and these genes are often expressed in cancer. What we think is that this pathway is remarkably preserved in most cancers.

Elizabeth McKenna: Can you tell us about efforts to try to drug or treat or target autophagy?

Dr. White: There's a lot of interest in that, and I think their initial attempts involve using hydroxychloroquine, which is a lysosomotropic drug. What happens is that the autophagy pathway captures the cargo and delivers it to the lysosome for degradation. The idea is that if you interfere with the lysosome, you're going to block all lysosome-dependent degradation including that which goes through the autophagy pathway. Hydroxychloroquine is involved in clinical trials to see if interfering with lysosome function might have some anticancer activity. Some of the early results from these trials have been published and are mildly encouraging. Other pharmaceutical companies are making deliberate inhibitors of specific components of the autophagy pathway. There could be something that's more specific other than just targeting the lysosome.
Elizabeth McKenna: Are there specific combination approaches that you foresee might be a good idea based on the literature?

Dr. White: Autophagy generally is induced in response to stress. Not just starvation, but pretty much any kind is stress. There are many papers published that say that autophagy is induced by just about any type of cancer treatment. One possibility is that anything that's damaging or stressful to cells might benefit from combination with autophagy inhibitors. Anything that's interfering with metabolism would probably be predicted to be greatly enhanced by inhibiting autophagy, because autophagy might be a rescue mechanism.

Elizabeth McKenna: Can you talk at all about some of the links between abnormal metabolism in cancer and autophagy? Are there certain triggers or certain pathways that are especially linked to autophagy?

Dr. White: What we've learned is that when autophagy degrades substrates and uses them to support metabolism, the key pathways appear to involve the mitochondria. The substrates are delivered to the mitochondria. They enter in the TCA cycle and keep it going. The TCA cycle maintains mitochondrial respiration and also generates aspartate, for example, to provide carbon for nucleotide synthesis. Everything that we've been able to see is related to autophagy providing substrates for mitochondrial metabolism to maintain respiration and energy homeostasis and to provide carbon for de novo nucleotide synthesis.

Elizabeth McKenna: The field of autophagy has been really hot lately. Where do you see the field going? What do you think the next big unanswered questions are?

Dr. White: I think it would be the connection between autophagy and the immune system. What we have seen, and many other people have also seen, is that when you inactivate autophagy in cancer, you promote an immune response to those tumors. In lung cancer, this is so dramatic that when you have autophagy-deficient lung tumors, even though they're small and benign, the inflammatory response of these tumors is so great that the mice die of pneumonia. It could be that what's happening is you've got sick tumor cells, you've got dead tumor cells, and the immune system is doing what it's supposed to do: go in there and clean things up. With the advances of immune therapy, this presents an intriguing question as to whether autophagy inhibition is going to be an advantage in combination with immune checkpoint inhibition or in immune therapy where $\mathrm{T}$ cells are activated to kill tumor cells.

Elizabeth McKenna: Does autophagy play a role in the immune cells themselves?

Dr. White: I think the answer is yes, but the open question is, which comes first? If autophagy is dispensable in the short term for immune cell function, then that doesn't matter. 


\section{$\$_{\text {CSH\& }}^{\infty}$ Cold Spring Harbor Symposia SYMPOSIA}

\section{A Conversation with Eileen White}

Cold Spring Harb Symp Quant Biol 2016 81: 360-361 originally published online January 25, 2017

Access the most recent version at doi:10.1101/sqb.2016.81.031591

$\begin{aligned} \begin{array}{r}\text { Creative } \\ \text { Commons } \\ \text { License }\end{array} & \begin{array}{l}\text { This article is distributed under the terms of the } \\ \text { http://creativecommons.org/licenses/by-nc/4.0/, which permits reuse and } \\ \text { redistribution, except for commercial purposes, provided that the original } \\ \text { author and source are credited. }\end{array} \\ \begin{array}{c}\text { Email Alerting } \\ \text { Service }\end{array} & \begin{array}{l}\text { Receive free email alerts when new articles cite this article - sign up in } \\ \text { the box at the top right corner of the article or click here. }\end{array}\end{aligned}$

To subscribe to Cold Spring Harbor Symposia on Quantitative Biology go to: http://symposium.cshlp.org/subscriptions 